\section{IMPACT OF PATENT DUCTUS ARTERIOSUS (PDA) ON POSTNATAL GROWTH AND COGNITION OF VERY LOW BIRTHWEIGHT INFANTS}

Le Ye Lee, Tammy SH Lim, Michelle LN Tan, Marion Margaret Aw, Krishnamoorthy Najduvae, Evelyn C Law. Singapore

\subsection{6/bmjpo-2021-RCPCH.162}

Background The management of a hemodynamically significant patent ductus arteriosus (hsPDA) associated with cardiomegaly and at least more than $1.5 \mathrm{~mm}$ in preterm infants is still a controversial topic. Previously, prophylaxis with ibuprofen was practiced in some units Nowadays, a conservative strategy with the use of diuretics and fluid restriction is considered a treatment option, alongside medical treatment with cyclo-oxygenase inhibitors (eg. Ibuprofen,indomethacin), paracetamol and surgery. The impact of the these practices have not been well studied.

Objectives We aim to determine the impact of hsPDA on the short term growth and cognitive outcomes of very low birth weight (VLBW) infants managed at a tertiary unit in Singapore from 2014 to 2018.

Methods A retrospective study on growth and cognitive outcomes was conducted on patients recruited from the outpatient follow-up clinic. Baseline neonatal demographics, treatment of hsPDA and short term neonatal morbidities were collected. Information on postnatal growth, health outcomes and cognitive testing scores were collected as well. Categorical data were compared with Chi squared test between those with medical or surgery treatment and those with conservative treatment.Continuous data with normal distribution was reported with means and Student $t$ test was used for analysis. Those with skewed distribution was reported with medians and Mann-Whitney test was performed.

Results 89 children were recruited at a median age of 34.5 (IQR 18-52) months, with equal gender distribution. Interestingly, infants $(n=61)$ who had hsPDA requiring treatment were more mature (29.9 vs 27.5 weeks) and heavier $1305 \mathrm{~g}$ vs $985 \mathrm{~g}(\mathrm{P}<0.01)$ at birth compared to VLBWs with conservative treatment $(n=21)$. Despite their maturity and also larger weight, those who required treatment were more likely to have been mechanically ventilated $66.7 \%$ vs $33.3 \%$ ( $p<0.01)$. As such, those who required PDA treatment had increased incidence of chronic lung disease (CLD) at 36 weeks $(83.8 \%$ vs $60.8 \% \%, \mathrm{p}=0.04)$, increased length of stay (79.5 vs 59.0 days, $\mathrm{P}<0.01$ ) and requirement of inhaled medications on follow-up. Growth failure of VLBWs with conservative treatment was evident until 6 months corrected age. Median cognitive scores were not statistically different, 97(IQR 85-105) vs 92 (IQR 81-101) when measured at ages 2-5 years. No differences were seen in the hearing loss or cerebral palsy rates. No association between PDA treatment and CLD were identified with a low cognitive score of less than 85 .

Conclusions In our small cohort, the presence of a hemodynamically significant PDA requiring treatment was associated with a higher incidence of CLD needing long term medication but no impairment of cognition and growth at follow-up.

\section{EVALUATION OF RASBURICASE USE WITHIN THE PAEDIATRIC INTENSIVE CARE UNIT}

Alex Christmas, Elizabeth Henderson, Edgar Brincat. UK

\subsection{6/bmjpo-2021-RCPCH.163}

Background Tumour Lysis Syndrome (TLS) is an oncological emergency caused by the rapid lysis of tumour cells. One of the biochemical abnormalities seen is a high urate level; rasburicase, a recombinant urate oxidase, can therefore be employed in both prophylaxis and treatment. Studies show significant variability in treatment regimens; its use is based on guidelines, such as those from The British Committee for Standards in Haematology (BCSH). It also carries a small risk of haemolytic anaemia, especially in those with a G6PD deficiency.

Objectives This study aimed to evaluate rasburicase use within a PICU setting. We assessed whether our unit was following best practice in terms of treatment duration, drug dosage and frequency, and monitoring (both for treatment response and complications).

Methods This was a retrospective study of patients receiving treatment with rasburicase in our PICU from January 2015 to May 2020. Patients receiving rasburicase were identified using electronic prescribing records. Data concerning demographics, diagnosis, clinical condition, rasburicase administration, and monitoring was then obtained from Metavision and Orion Clinical Portal. TLS risk was determined retrospectively and children classed as either having High Risk Disease (HRD), Intermediate Risk Disease (IRD), or Low Risk Disease (LRD).

Results 21 patients receiving rasburicase were identified (18 with haematological malignancies, 3 with solid tumours). Data on ethnicity was available for $15 / 21$ children, all of whom were Caucasian. Ages ranged from 9 weeks to 16 years. 5/21 had evidence of lab TLS, with 4 of these meeting the criteria for clinical TLS. 11 children were assessed to have HRD, 5 as having IRD, and 5 as having LRD. All children who had features of TLS were at high risk. 9/21 children (42.9\%) received more than the 5-7 days treatment recommended by manufacturers and the $\mathrm{BCSH}$, with a range from 1-21 days. Most children received $0.2 \mathrm{mg} / \mathrm{kg}$ doses with only one child receiving a dose of $2 \mathrm{mg} / \mathrm{kg}$ on one occasion. 3 children received twice daily dosing. One child died after only one dose of rasburicase; of the remainder, $100 \%$ received daily FBCs, LFTs, and U\&Es while receiving rasburicase, while only $16 / 20$ (80\%) received daily urate levels to monitor response. No cases of haemolytic anaemia due to rasburicase therapy were documented, but $17 / 21$ children required at least one red cell transfusion whilst a PICU inpatient. G6PD status was checked in only one of the children.

Conclusions Significant variability in use of rasburicase exists within our PICU. Duration of rasburicase treatment varies widely and several other practices outwith those recommended by current best practice were observed, such as its use in children at low risk of developing TLS, repeated treatment courses, twice daily doses, and a child who received a $>0.2$ $\mathrm{mg} / \mathrm{kg}$ dose. Active monitoring for haemolytic anaemia outwith daily FBCs is limited and is complicated by the frequency of children requiring blood transfusions. Awareness of this as a serious complication in a small number of children may need to be raised. 